\title{
Effects of the Three-Dimensionality of Ship Hull on the Wave Impact Pressure
}

\author{
Iwao Watanabe*, Member
}

\begin{abstract}
Summary
A general expression for the impact pressure distribution on the flat bottom ship making slamming motion in waves is derived by matched asymptotic expansion on the bases of the shallow draft and the gravity free assumptions. 3-D flat plate formulation is utilized to have the solution in the outer region. For the solution in the inner region, its flow similarity to 2-D planing plate flow of which solution is known analytically is made use of. Both solutions are matched asymptotically to make them determinate and a composite solution valid in the entire region is obtained. The calculated results are compared with the experiments done by using free running model of a bulk carrier. Agreements between the experiments and the estimation based upon the OSM ship motion data are not satisfactory as expected. The cause of the discrepacy has been examined to find that it should be attributed to lack of the accuracy of the OSM estimation rather than the present pressure formula. It is shown that the situation can be improved considerably by using measured ship motion data instead of the OSM data.
\end{abstract}

\section{Introduction}

The wave impact pressure has been a crucial subject to the design of the ship structure since early days of the naval architecture. Naturally, there have been lots of works on this matter. Among them, von Kármán or Wangner's theories ${ }^{112)}$ have been used for the forces and the pressure assuming that the impact phenomena are two dimensional in the transverse section. And their theories are known to give good estimation for the most hull forms if no air entrapping is involved. There arise, however, some difficulties in calculating impact pressure for the actual cases. Namely, for hull sections having the flat bottom, these theories predict infinite impact pressure because the waterline varies at infinite rate in the two dimensional sense. Of course this is not the case in reality. Existing remedies for it is to introduce empirical pressure in connection with local bottom configuration ${ }^{3}{ }^{4)}$. It seems to the author that the sound treatment should comprise of due consideration to the three dimensionality of the field. Theoretical researches on this aspect are rather scarce and an axisymmetric case is examined $^{5)}$ so far to this author's knowledge.

Recently, the present author has worked on the three-dimensionality of water entry problem and shown that estimation of the pressure can be improved by taking into account of the effects of

* Ship Research Institute the longitudinal flow along the hullif), . This method can be extended to include the wave impact in the same way since the problem will be reduced to the contact problem between the hull and the water surface. This paper discusses the wave impact pressure both theoretically and experimentally when the bottom surface hits the wave surface.

\section{Theoretical consideration}

Let us take a case where the ship hits into the surface of incoming wave with heaving and pitching motions under way, as is shown in Fig. 1. The head sea is assumed. The ship is assumed to have forward velocity $U$.
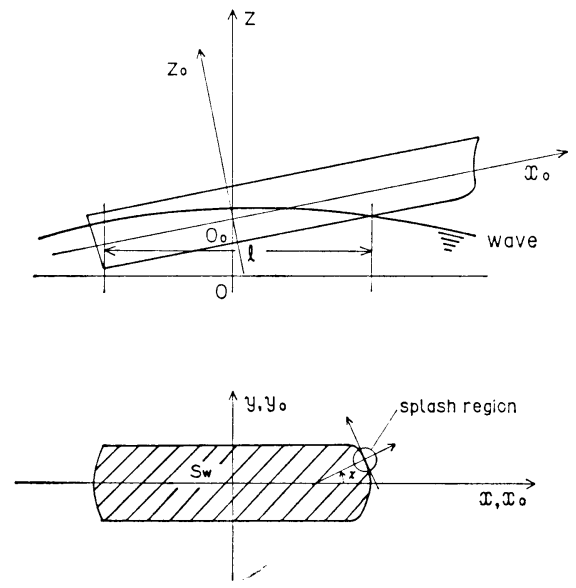

Fig. 1 Coordinate system 


\section{1 Basic formulation}

The ship is assumed to have very shallow draft compared to the horizontal dimensions of the waterplane during the motion. The assumption may be applicable to the impact of the forward bottom.

Although basic formulation adopted in the water entry problem can be applied in principle, some revision is needed since the normal velocity distribution at the bottom can be more complex in the case of the wave impact problem than that of the water entry in still water.

It is assumed a priori that the gravity free assumption holds and the free surface condition can be replaced by equi-potential condition as in the waterientry problem for convenience. This will be justified since the fluid is accelerated at much greater rate than that of gravity.

Let the equation of the hull in the body fixed coordinate $\left(x_{0}, y_{0}, z_{0}\right)$ be

$$
z_{0}=\varepsilon f\left(x_{0}, y_{0}\right)
$$

We will take space fixed coordinate $(x, y, z)$. The relation between the both coordinates is

$$
\left.\begin{array}{l}
x=x_{0}+U t \\
y=y_{0} \\
z=z_{0}-h-x_{0} \psi
\end{array}\right\}
$$

where pitching $(\psi)$ and heaving $(h)$ are defined with respect to the origin of the coordinate. Bow down rotation and downward translation are taken positive direction for respective modes of motion.

The equation of the hull surface with respect to space fixed coordinate system is

$$
F(x, y, x, t)=z+h+(x-U t) \psi-\varepsilon f(x-U t, y)=0
$$

The boundary condition on the hull moving in the incoming waves is

$$
\nabla F \cdot \nabla\left(\Phi+\Phi_{w}\right)=-F_{t},
$$

where $\Phi$ denotes perturbed velocity potential and $\Phi_{w}$ denotes velocity potential of the incoming wave. Suffix $t$ stands for the differentiation with respect to $t$. The latter potential can be written as

$$
\Phi_{w}=-g \zeta_{0} / \omega \exp (k z) \sin (k x+\omega t)
$$

The wave elevation and the wave slope are written respectively as

$$
\begin{aligned}
& \zeta_{w}=\zeta_{0} \cos (k x+\omega t) \\
& \theta_{w}=-k \zeta_{0} \sin (k x+\omega t)
\end{aligned}
$$

Substituting them into the boundary condition, we have

$$
\begin{array}{r}
\nabla F \cdot \nabla \Phi=\nabla F \cdot \nabla \Phi_{w}-h_{t}-x_{0} \psi_{t}+U\left(\psi-\varepsilon f_{x}\right) \\
\text { on } F(x, y, z, t)=0
\end{array}
$$

where

$$
\nabla F=\left(\psi-\varepsilon f_{x},-\varepsilon f_{y}, 1\right)
$$

It is seen that the problem is reduced to solve the unsteady flow field around the deforming body.

\subsection{Shallow draft assumption}

Because of the shallow draft assumption, the horizontal components can be neglected since they are much smaller than the vertical component except edge regions where impact phenomena take place. The boundary condition can be placed on the $z=0$ surface instead of the actual hull surface. Thus, the hull surface condition is linearized as follows

$$
\Phi_{z}=-\Phi_{w_{z}}-\left(h_{t}+x_{0} \psi_{t}\right)+U \psi \text { on } z=0 \text { and } S_{w}
$$

The wateplane $S_{w}$ is defined as the solution of

$$
h(t)+x_{0} \psi(t)=\varepsilon f(x, y)
$$

and $S_{w}$ is time-dependent naturally.

Generally speaking, the waterplane during the motion will expand in the rear and sides as well as in the frontal direction. We will assume that the expansion in the rear and the sides is too small to be related to the occurrence of the impact. This means the waterplane of the ship runs forward and the impact occurs there. We call this region splash region since the impact accompanies the occurrence of the splash.

This linearization is violated in the splash region since the horizontal velocity terms discarded in (10) become much bigger order than 0(1). Another expansion is necessary to formulate the edge region. It will be discussed in a later section.

\section{3 Integral equation}

It is easy to see that the boundary condition (10) has a quite similar form to the water impact case, except for the spatial dependence with respect to $x$ direction arising from $\Phi_{w z}$. Therefore the flat plate formulation used for the water impact problem can be utilized with a slight modification. Let $\mu(x, y)$ be the doublet distribution over the plate $S_{w}$. Then the velocity potential is expressed by

$$
\Phi=\int_{S_{w}} \mu(\xi, \eta) \frac{\partial}{\partial z}\left(\frac{1}{R}\right) d s
$$

where

$$
\begin{aligned}
& R=\sqrt{ }(x-\xi)^{2}+(y-\eta)^{2}+z^{2} \\
& \mu\left(\xi, \gamma_{i}\right)=(\Phi(z=0+)-\Phi(z=0-)) / 4 \pi \\
& \quad=-\Phi(z=0-) / 2 \pi
\end{aligned}
$$

The strength of the doublet distribution is determined as the solution of an integral equation which states that the induced velocity by the doublet is equal to the normal velocity on the plate.

$$
\begin{aligned}
v(x, y) & =\lim _{z \rightarrow 0} \frac{\partial \Phi}{\partial z}=\lim _{z \rightarrow 0} \int_{S_{w}} \mu(\xi, \eta) \frac{\partial^{2}}{\partial z^{2}}\left(\frac{1}{R}\right) d s \\
& =f_{S_{w}} \frac{\mu(\xi, \eta)}{\left\{(x-\xi)^{2}+(y-\eta)^{2}\right\}^{3 / 2}} d s, \\
& =f_{-b}^{b} \int_{0}^{l(\eta)} \frac{\mu(\xi, \eta)}{\left\{(x-\xi)^{2}+(y-\eta)^{2}\right\}^{3 / 2}} d \xi d \eta,
\end{aligned}
$$

$v(x, y)$ is given by Eq. (10). The second part of the integral is singular, of which finite part is defined by 


$$
\begin{aligned}
f_{S_{w}} & \frac{\mu(\xi, \eta)}{\left\{(x-\xi)^{2}+(y-\eta)^{2}\right\}^{3 / 2}} d s \\
= & \int_{S_{w}} \frac{\mu(\xi, \eta)-\mu(x, y)}{\left\{(x-\xi)^{2}+(y-\eta)^{2}\right\}^{3 / 2}} d s \\
& +\mu(x, y) \lim _{z \rightarrow 0} \int_{S_{w}} \frac{1}{\left\{(x-\xi)^{2}+(y-\eta)^{2}+z^{2}\right\}^{3 / 2}} d s
\end{aligned}
$$

\section{4 Numerical solution}

We will look for the strength of the distribution numerically. First of all, the characteristics of the flat plate will be examined. It is common feature for the plate to have a very narrow breadth compared with the longitudinal scale and to be symmetric horizontally. Thus we will assume that the flat plate considered here is slender and symmetric about the centerline and has almost constant longitudinal length and has invariant breadth $(b)$ irrespective of time.

According to the standard scheme of the aerofoil theory, the strength of the doublet distribution is assumed in polynomial function as

$$
\mu(\xi, \eta)=(V / b) \sqrt{ } \xi(l-\xi) \sqrt{ } b^{2}-\eta^{2} \sum_{n=0}^{N-1} A_{n}(\xi-l / 2)^{n} .
$$

where $V$ is an appropriate characteristic velocity in the vertical direction and

$l=l(\eta)(-b<\eta<b)$ is longitudinal length of the waterline.

Since the flat plate is slender, the flow in the transverse direction can be approximated by simple flow which has been expressed by elliptic loading. Thus we have

$$
\begin{aligned}
v(x, y)= & (V / b) \sum_{n=0}^{N-1} A_{n} \int_{-b}^{b} \sqrt{ } b^{2}-\eta^{2} \int_{0}^{l(\eta)} \\
& \frac{(\xi-l / 2)^{n}}{\left\{(x-\xi)^{2}+(y-\eta)^{2}\right\}^{3 / 2}} \sqrt{\xi(l-\xi)} d \xi d \eta
\end{aligned}
$$

\subsubsection{Equivalent waterplane}

The equation which determines the unknowns can be set up by taking the same number of the control points as that of the unknowns and by evaluating (17) at respective points. Since the only one mode of variation is assumed in the $y$ direction, a control point in $y$ direction is fixed to $y=0$. Thus $N$ locations along the centerline are taken as the control points.

Special care is needed for the numerical evaluation of a singular integral. Moreover the above integral has a form that the $\xi$ integration has to be done first, which makes integration numerically twice. We will try to simplify the integration by introducing the "equivalent waterplane" concept.

The assumption that the variation of waterline length in the $\eta$ direction is so little allows us to treat the fore part of the waterline as straight line. Thus we will introduce rectangular plane of breadth $2 b$ called "equivalent waterplane", of which length is defined by

$$
l_{e}=S_{w} /(2 \mathrm{~b}) \text {, }
$$

where $S_{w}$ is the waterplane area enclosed by Eq. (11).

Because of the additional assumption, double integration will become interchangeable. If we integrate in the $\eta$ direction, the result will be given in the analytical form. After some algebra, we get the equation to give $B_{n}$ 's as

$$
\begin{aligned}
& v\left(x_{i}, 0\right)=2 \lambda_{e} \sum_{n=0}^{N-1} B_{n}\left[-G_{n}^{1}\left(x_{i}\right)+G_{n}^{2}\left(x_{i}\right)\right], \\
& i=1, N
\end{aligned}
$$

where

$$
\begin{aligned}
& B_{n}=A_{n}\left(l_{e} / 2\right)^{n} \\
& G_{n}{ }^{1}=\int_{-1}^{1} \frac{K\left(k^{2}\right)}{\left\{(x-\xi)^{2}+r^{2}\right\}^{1 / 2}} \xi^{n} d \xi, \\
& G_{n}{ }^{2}=\int_{-1}^{1} \frac{E\left(k^{2}\right)}{(x-\xi)^{2}} \xi^{n} \sqrt{ } 1-\xi^{2} \sqrt{ }(x-\xi)^{2}+r^{2} d \xi \\
& \text { and } \quad r=2 b / l_{e} .
\end{aligned}
$$

The integrals can be reduced to simpler forms for numerical evaluation by extracting singular terms out of the original form, integrating them analytically and putting together all of them,

$$
\begin{aligned}
G_{n}{ }^{1}= & \int_{-1}^{1}\left[\frac{K\left(k^{2}\right)}{\left\{(x-\xi)^{2}+r^{2}\right\}^{1 / 2}} \xi^{n}-x^{n} \frac{\sqrt{1-x^{2}}}{r} \times\right. \\
& \left.\ln \frac{4 \sqrt{ }(x-\xi)^{2}+r^{2}}{|x-\xi|}\right] d \xi+x^{n} \frac{\sqrt{1-x^{2}}}{r}[(1-x) \times \\
& \ln \frac{4 \sqrt{ }(\overline{x-1})^{2}+r^{2}}{|x-1|}+(1+x) \ln \frac{4 \sqrt{(x+1)^{2}+r^{2}}}{|x+1|} \\
& \left.+(r / 2)\left\{\tan ^{-1}\left(\frac{1-x}{r}\right)+\tan ^{-1}\left(\frac{1+x}{r}\right)\right\}\right] \\
G_{n}{ }^{2}= & \int_{-1}^{1} \frac{H(\xi)-H(x)-H_{1}(x) X-H_{2}(x) X^{2}}{\times \ln \left\{\left(X^{2}+r^{2}\right) / X^{2}\right\}} \\
& -H(x)\left\{\frac{1}{1-x}+\frac{1}{1+x}\right\}+H_{1}(x) \ln \left(\frac{1-x}{1+x}\right) \\
& +H_{2}(x)\left[(1-x) \ln \frac{\sqrt{ }(x-1)^{2}+r^{2}}{|x-1|}\right. \\
& +(1+x) \ln \frac{\sqrt{ }(x+1)^{2}+r^{2}}{|x+1|}+(r / 2) \\
& \left.\times\left\{\tan ^{-1}\left(\frac{1-x}{r}\right)+\tan ^{-1}\left(\frac{1+x}{r}\right)\right\}\right],
\end{aligned}
$$

where

$$
\begin{aligned}
& H(\xi)=\sqrt{ } x^{2}+r^{2} \sqrt{1-\xi^{2}} \xi^{n} E\left(k^{2}\right), \\
& H(x)=r x^{n} \sqrt{1-x^{2}} \\
& H_{1}(x)=n r x^{n-1} \sqrt{1-x^{2}}-\frac{r x^{n+1}}{\sqrt{1-x^{2}}} \\
& H_{2}(x)=\frac{x^{n}}{2 r} \sqrt{1-x^{2}}, \\
& X=\xi-x
\end{aligned}
$$

2.4.2 Pressure distribution

Once the integral equation is solved and the doublet distribution is derived, the pressure can be obtained by differentiating the velocity potential 
with respect to time. From Bernoulli's equation, we have the surface pressure distribution as

$$
\begin{aligned}
& \frac{p}{\rho}(z=0)=-\frac{\partial \Phi}{\partial t}(z=0)=2 \pi \frac{\partial \mu}{\partial t} \\
& =2 \pi(V / b) \frac{\partial}{\partial t} \sqrt{\xi(l-\xi)} \sqrt{ } b^{2}-\eta^{2} \sum_{n=0}^{N-1} A_{n}(\xi-l / 2)^{n} \\
& =\frac{V^{2}}{2}\left[u K(x, y)\left(\frac{x}{l-x}\right)^{1 / 2}+2(1 / V) \sqrt{x(l-x)} \frac{\partial K}{\partial t}\right]
\end{aligned}
$$

where

$$
\begin{aligned}
& u=\frac{1}{V} \frac{\partial l}{\partial t} \\
& K(x, y)=(2 \pi / b) \sqrt{ } b^{2}-\eta^{2} \sum_{n=0}^{N-1} A_{n}(\xi-l / 2)^{n}
\end{aligned}
$$

The second term contributes little to the peak value of the pressure. We have asymptotic behavior of the distribution near the splash region,

$$
\frac{p}{\rho} \rightarrow \frac{V^{2}}{2}\left\{u K(l, y)\left(\frac{l}{l-x}\right)^{1 / 2}\right\}
$$

It is seen that the pressure becomes singular as approaching to the region in the order of square of the distance and monotonically diverges without any peak. This is the limitation of the linear solution. Another solution which compensate this deficiency will be sought in the following.

\section{5 Numerical example of outer solution}

Before proceeding to the derivation of the splash region solution, let us examine how the flow over the bottom surface is expressed in the outer solution. As the form of the boundary condition shows clearly, it can be decomposed to the codition due to heaving, the condition due to pitching and that of the incoming wave. In other words, the unknown constants $B_{n}$ for the heaving, pitching and incoming wave can be determined independently and superposed afterward to give a solution for any combination of motions and wave. We will discuss nature of $B_{n}$ for several modes separately.

Fig. 2 shows how $N$, the total number of terms affects $B_{n}$ 's of heaving mode. It is seen that

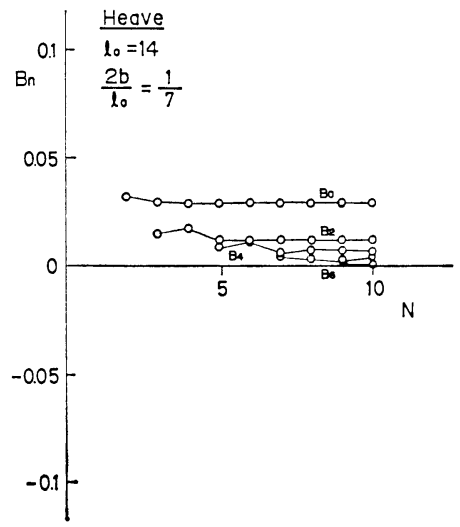

Fig. 2 Variation of $B_{n}$ to $N$
$B_{0}$ is dominant and all $B_{n}$ keep almost constant value while $N$ is increased. This indicates that the polynomial is stable and is able to give good approximation of the distribution with a few terms. Also shown is the variation of $B_{n}$ to the length breadth ratio in Fig. 3 . As the model is getting slender, the higher order terms become significant. This reflect the fact that the relatively abrupt change in the splash region of more slender body requires higher order modes of variation. The dotted line in the figure shows the sum of $B_{n}$ which is an index of the extent of the pressure variation in the edge region. The sum becomes small as the model is getting slender.

Fig. 4 shows the variation of $B_{n}$ to $N$ in the case of the incoming wave when the wave is

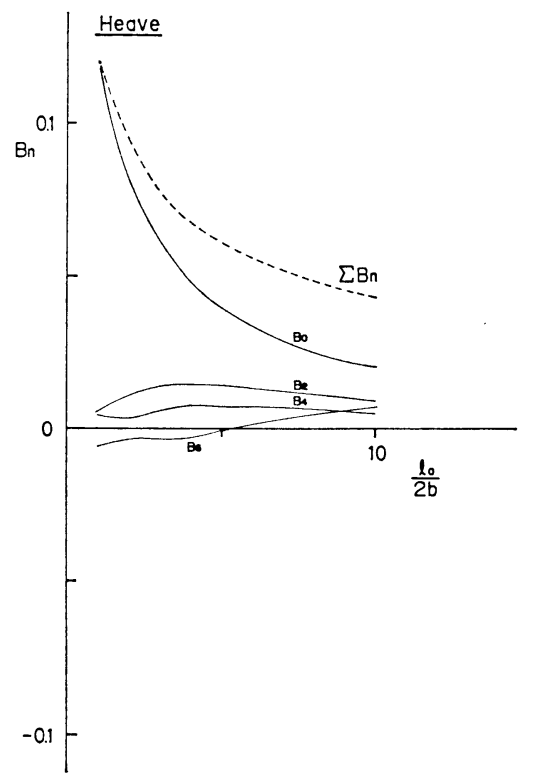

Fig. 3 Variation of $B_{n}$ to length breadth ratio

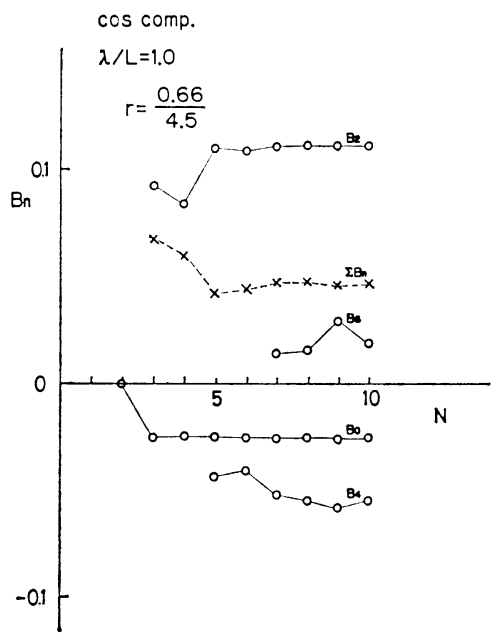

Fig. 4 Variation of $B_{n}$ to $N$ 


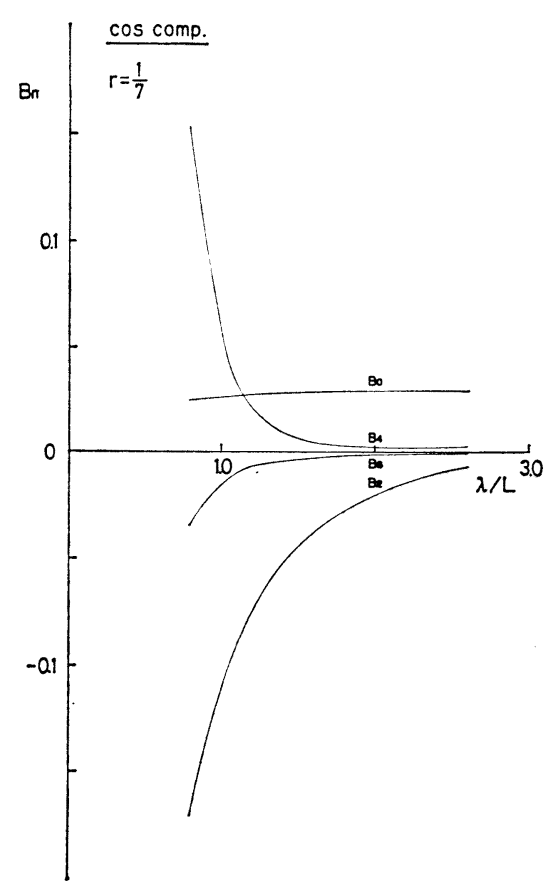

Fig. 5 Variation of $B_{n}$ to wave length

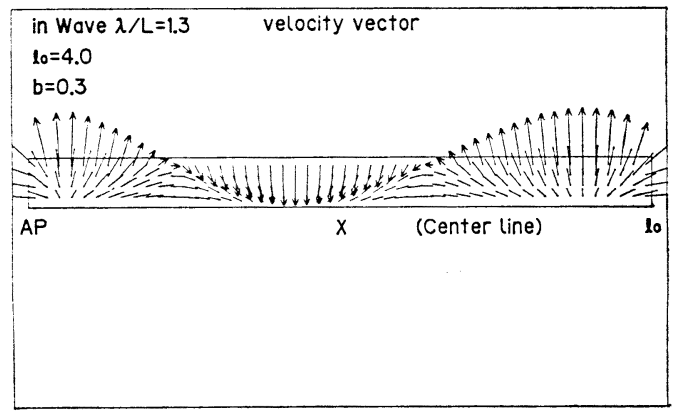

Fig. 6 Horizontal velocity over the bottom surface

in cosine phase with respect to the midship. It is seen that they are in stable condition against changing $N$, as was seen in heaving problem. Next we will see how these $B_{n}$ varies to the wave length of incoming wave. Fig. 5 shows that the shorter the wave length becomes, the larger $B_{n}$ become. The terms greater than $B_{O}$ are attenuated rapidly as the wave is getting longer. This trend is in accordance with heaving problem.

\subsubsection{Velocity distribution}

Distribution of horizontal velocity over the bottom surface is shown in Fig. 6. The bottom is assumed flat plate and the model in the wave of $\lambda / L=1.3$ is making motions estimated by OSM. It is seen that the horizontal velocity changes its pattern drastically due to the phase difference between incoming wave and the motion.

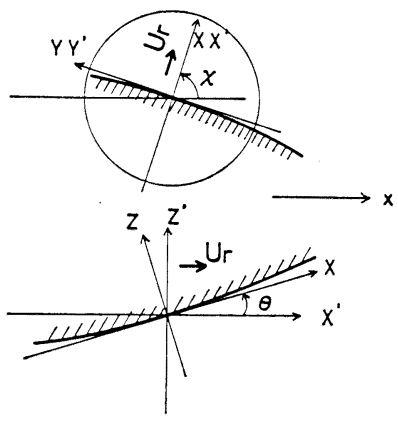

$S=X+i Z$

Fig. 7 Coordinate system in the splash region

\section{6 Singular region}

The flat ship assumption is violated in the splash region where the wave impact occurs. Since the velocity potential given by the flat plate solution approaches zero as order of square root of the distance from the edge, its $x$ and $y$ derivatives, $\Phi_{x}$ and $\Phi_{y}$ diverge as inverse square root of the distance. It is seen that the singular region is within $\varepsilon^{2}$ of the edge. It has been show $n^{6 \sim 8)}$ that, if we introduce inner expansion over there and reformulate the boundary condition, the flow over there can be approximated by the flow pattern near the stagnation point of the flat plate at the inclined position to the free stream. Details in the formulation of the boundary value problem and the reduction of the analytical expression on the velocity potential will not be repeated here.

Let $S$ be inner coordinate of complex number shown in Fig. 7. $S$ becomes real on the body surface. The resulting surface velocity over the plate is

$$
\frac{u}{U_{r}}=\frac{1+\nu(S / \Delta)-\sqrt{(1+\nu)(S / \Delta+c)}}{-(S / \Delta)},
$$

where $\nu=\cos \theta$.

Pressure distribution is given by

$$
C_{p}=\frac{u^{2}}{p U_{r}^{2} / 2}=\frac{2 D}{2 D+(S / \Delta)^{2}},
$$

where $D=k^{2}(S / \Delta+c)+k(1+\nu S / \Delta) \sqrt{S / \Delta+c}$,

$$
k=\sqrt{1+\nu} \text { and } c=\frac{1}{1+\nu} \text {. }
$$

\section{7 Composite expansion}

We now have both outer and inner solutions which are valid in the respective regions. Two solutions, after adding and subtracting the common part, will be combined to give forth a uniformly valid composite solution in the entire region as

$$
\begin{aligned}
C_{p c}= & C_{p 0}+C_{p \imath}-C_{p c o m} \\
C_{p c}= & \cot \theta\left[u K(x, y)\left(\frac{x}{l-x}\right)^{1 / 2}\right. \\
& +2(1 / V) \sqrt{x(l-x)} \frac{\partial K}{\partial t}+\cot \theta \frac{2 D}{2 D+(S / \Delta)^{2}}
\end{aligned}
$$




$$
\begin{aligned}
& \left.-u K(l, y)\left(\frac{x}{l-x}\right)^{1 / 2}\right], \\
& \text { where } \quad C_{p}=\frac{p}{p V^{2} / 2} \\
& D=k^{2}(S / \Delta+c)+k(1+\nu S / \Delta) \sqrt{ } S / \Delta+c \\
& S=\frac{(l-x) \cos \chi+\left(y-y_{b}\right) \sin \chi}{\varepsilon^{2}},
\end{aligned}
$$

$\theta$ is the angle between the wave surface and the hull in the normal direction of the waterline and $(\cos \chi, \sin \chi)$ is the normal vector to the waterline (refer to Fig. 1 and Fig. 7).

\section{8 Prediction of wave impact pressure}

It was shown so far that, if we can specify the boundary values on the bottom, the impact pressure in the waves can be calculated from the above formulation. This means that we can have a method to estimate impact pressure which was not possible in the existing method. Typical example of that problem is the pressure on the flat plate. Conventional two dimensional methods give infinite value of impact pressure.

In applying present method, we need ship motions and the relative wave motion. Ship motion program such as strip method can be utilized for that purpose. The relative velocity and the inclination at the impact can be reduced easily from the result. The details of the estimation goes as follows ;

(1) Calculate the ship motions. Pitching and the relative water speed at the forward bottom at the moment of the contact of the bottom to the water surface are essential.

(2) Determine the boundary condition from Eq. (10).

(3) Derive the outer solution by solving the integral equation.

(4) Calculate relative inclination from pitching angle, instantaneous wave slope, the trim angle and the local body slope.

(5) Obtain the matched asymptotic expression

\begin{tabular}{|c|c|}
\hline $\begin{array}{l}\text { in Wove } \lambda / L=1.3 \\
l_{0}=4.0 \\
b=0.3 \\
b=10^{\circ}\end{array}$ & pressure distribution $\left(C_{P}\right)$ \\
\hline AP & $x$ \\
\hline
\end{tabular}
of the impact pressure.

An example of the calculated impact pressure distribution over the bottom of the model ship

Fig. 8 Pressure distribution over the bottom surface is shown in Fig. 8. The model is assumed to have rectangular flat bottom and is making ship motions in the wave of $\lambda / L=1$. T. The trim angle is assumed $5 \mathrm{deg}$. It is seen that the pressure rises very sharply near the bow. The several lines seen in the distribution correspond to small difference in the distribution in the transverse direction. The pressure rises more sharply as the location goes to the sides.

\section{Experiment}

\section{1 Details of experiment}

Experiment was made so as to obtain data to check the validity of the present theory. A model is an elastic model of a medium-sized bulk carrier in the ballast condition. The principal particulars of the model are shown in Table 1 . The characteristics of the elastic model and the results on the elastic responses will not be discussed here since they were already presented elsewhere.

The experiment took place in the seakeeping basin of Ship Research Institute. The model was run in various kinds of waves with the different

Table 1 Principal particulars of a model

\begin{tabular}{|l|l|}
\hline$L(m)$ & 4.5 \\
\hline$B(m)$ & 0.659 \\
\hline$d_{f}(m)$ & 0.126 \\
\hline$d_{a}(m)$ & 0.226 \\
\hline$d_{m}(m)$ & 0.176 \\
\hline$G M(m)$ & 0.114 \\
\hline$\Delta(k g)$ & 413.9 \\
\hline$k_{y y}$ & $0.247 L$ \\
\hline
\end{tabular}
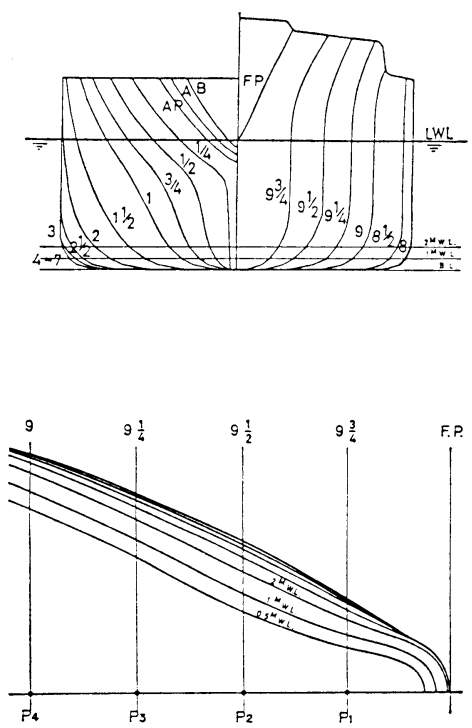

Fig. 9 Body plan, waterline in the forepart and the location of pressure gauges 
heading and forward velocity. During the experiment, measurements have been made on pressures over the forward bottom, together with pitching, rolling, vertical accelerations and the relative wave elevation at respective locations. Schematic body plan and the location of the pressure gauges are shown in Fig. 9.

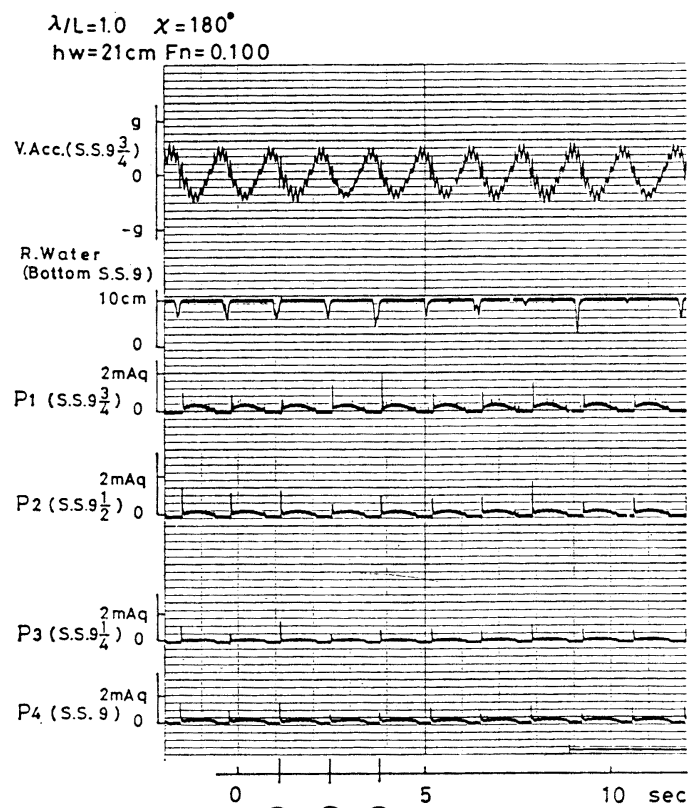

(a) (b) (C)

Fig. 10(1) Example of measured data

$$
\lambda / L=1.0 \quad \chi=180^{\circ}
$$$$
\text { hw } 21 \mathrm{~cm} \mathrm{Fn} 0.100 \text { Enlarged (a) }
$$

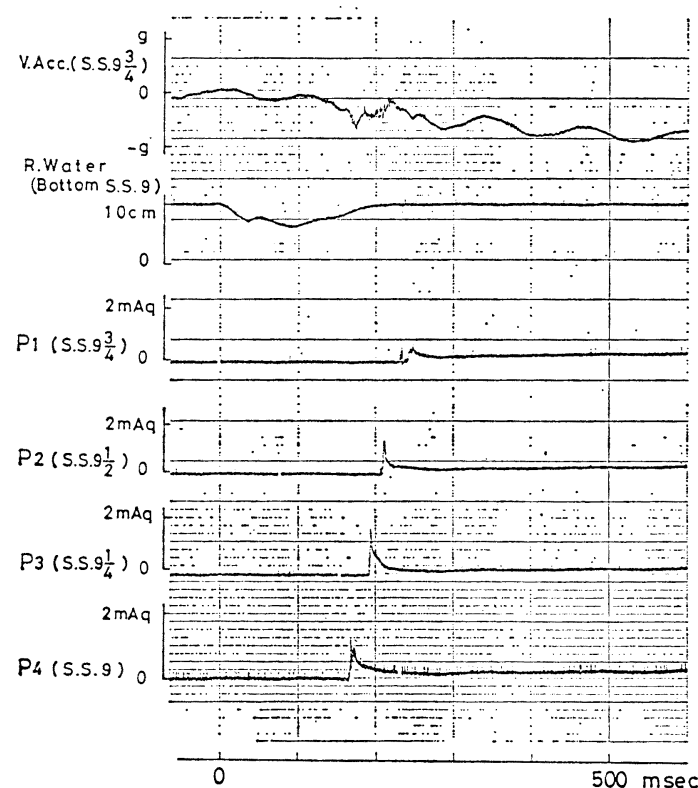

Fig. 10(2) Example of magnified measured data
Special attention has been paid to recording and analyzing the pressure data since they contain very high frequency components once the impact phenomena occurs. The casette tape recorder on board have been used instead of the radio telemetry to store analog signals of the pressure. This as-

$\lambda_{I L}=1.0 \quad \chi=180^{\circ}$

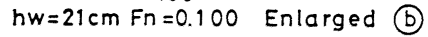

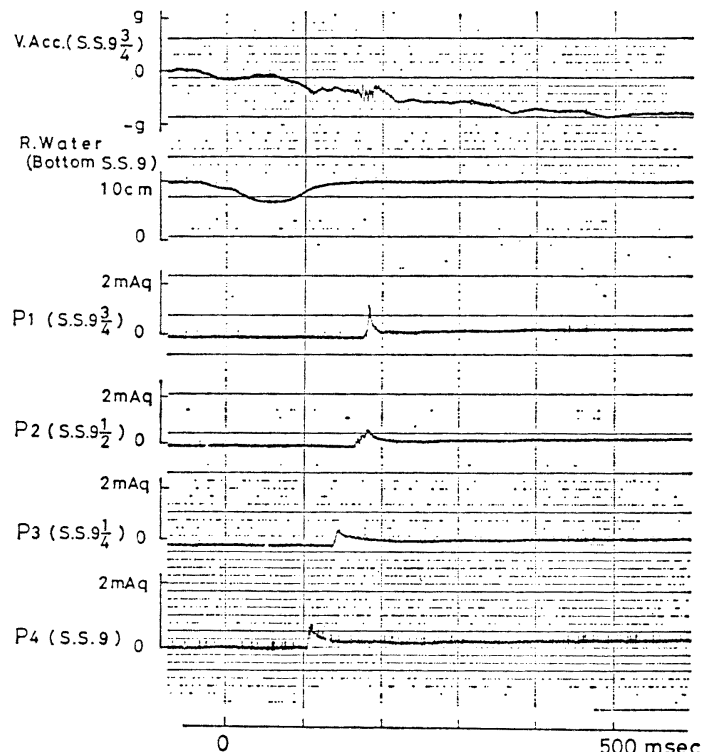

Fig. 10(3) Example of magnified measured data

$\lambda / L=1.0 \quad \chi=180^{\circ}$

$\mathrm{hw}=21 \mathrm{~cm} \mathrm{Fn}=0.100$ Enlarged (C)

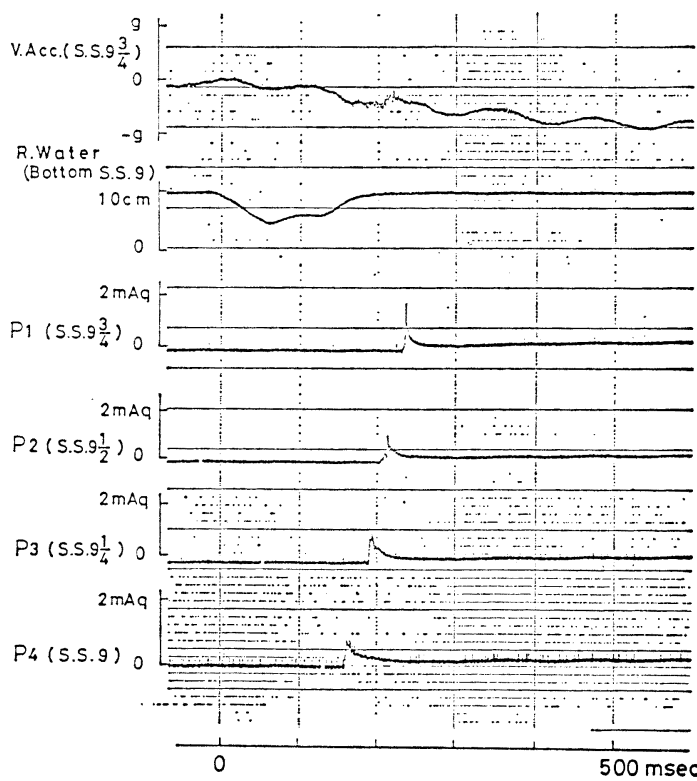

Fig. 10(4) Example of magnified measured data 
sures restoration of the high frequency components. The analyses have been made by utilizing the transient wave memory and high speed oscillograph so as to trace very sharp peak values of the impact pressure in detail.

\section{2 Experimental results}

An example of measured time histories is shown in Figs. 10-(1) through-(4). The figure with (1) are wave forms during an experiment while those with (2) through (4) show enlarged view at impact. Each figures presents, from top to bottom, vertical acceleration at the bow part (SS $81 / 2)$, relative water elevation at the bottom centerline of SS 9 (a wave gauge protruded from the bottom) and the pressures over the bottom. The pressure gauges are located along the bottom centerline at the SS $93 / 4$, SS $91 / 2$, SS $91 / 4$ and SS 9. The figure shows the result in the regular head sea condition of $\lambda / L=1.0$. The ship speed is about 0.1 in Froude number, as is shown in the figure. It is clear that the sharp impulsive peak runs toward the bow. The downward pulse in the relative wave shows that the bottom emergence took place at that moment. The relatively

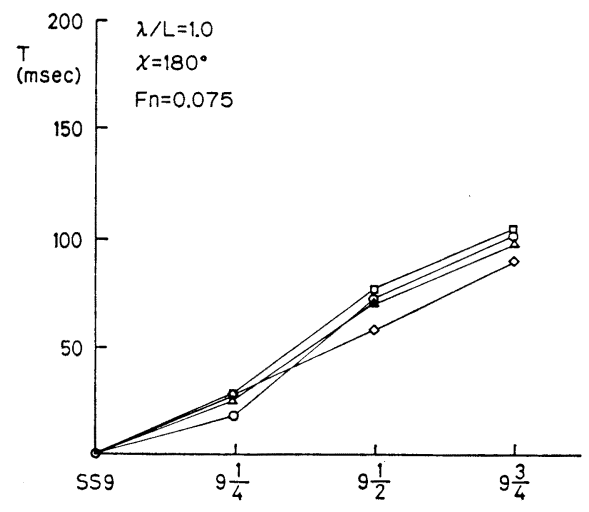

Fig. 11 Time lag in the occurrence of the impact $(\lambda / L=1.0)$

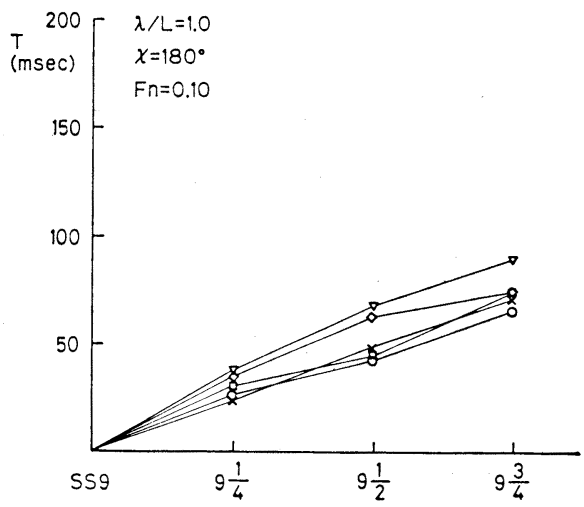

Fig. 12 Time lag in the occurrence of the impact $(\lambda / L=1.0)$ high frequency oscillation in the vertical acceleration is hull elastic vibration, which is typical feature of the elastic model.

The nature of impact can be characterized by its run, peak value and duration.

Let us examine run of impact defined by the velocity of the splash region. Fig. 11 to 14 show time lag when the impact pressure at consecutive pressure gauges are at their peak. The regular waves of $\lambda / L=1.0$ and 1.2 are taken. Several sequences out of one experiment are selected. The impact runs from aft to fore. It is also seen that the greater the forward speed becomes, the more the time lag decreases. This may be attributed to the fact that the ship motion tends to become severe as the forward velocity is increased.

The peak value of the bottom impact pressure will be examined next. Figs. 15 and 16 show the relation between the forward velocity and the peak pressures. The pressure is normalized with respect to the wave amplitude. The averaged values are shown in the figures. It is seen that, despite of the scattering of data, the greater the forward

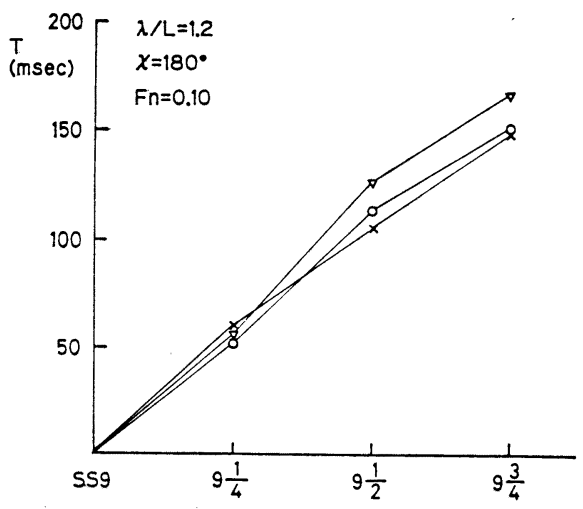

Fig. 13 Time lag in the occurrence of the impact $(\lambda / L=1.2)$

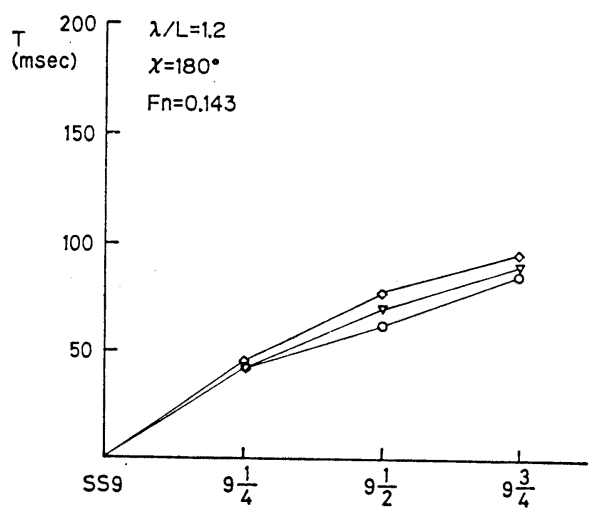

Fig. 14 Time lag in the occurrence of the impact $(\lambda / L=1.2)$ 

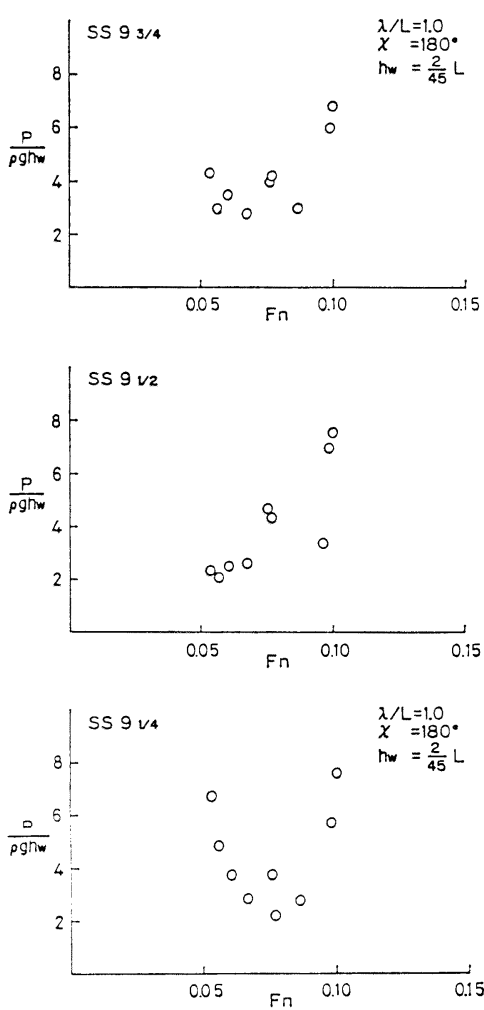

Fig. 15 Impact pressure versus Froude number $(\lambda / L=1.0)$

speed becomes, the more intense the impactipressure would be. It is also seen that the difference of the peak values with respect to the locations is not significant.

AS an index of duration of the impact, the time of duration $\left(T_{1 / 2}\right)$ during which the pressure stays above a half of the peak value is chosen. Fig. 17 shows the relation between $T_{1 / 2}$ and the bottom location. The data are taken out of several experiments. Although the scattering of the data is apparent, it is seen that the location affects little to the duration of impact and the average duration is around $5 \mathrm{msec}$.

\section{Comparisons}

It will be examined how well the present calculation can explain the experiment. The calculation was made on the pressure over the flat bottom, which is hard to estimate by conventional two dimensional calculation.

The motion data by OSM which is used as input in the pressure calculation will be examined first. Figs. 18 and 19 show the variation of pitching amplitude, the relative water elevation and phase difference between them versus forward velocity. By lines the estimated motion data are shown while the experiments are shown by symbols. Agreements in the amplitude are good as is seen
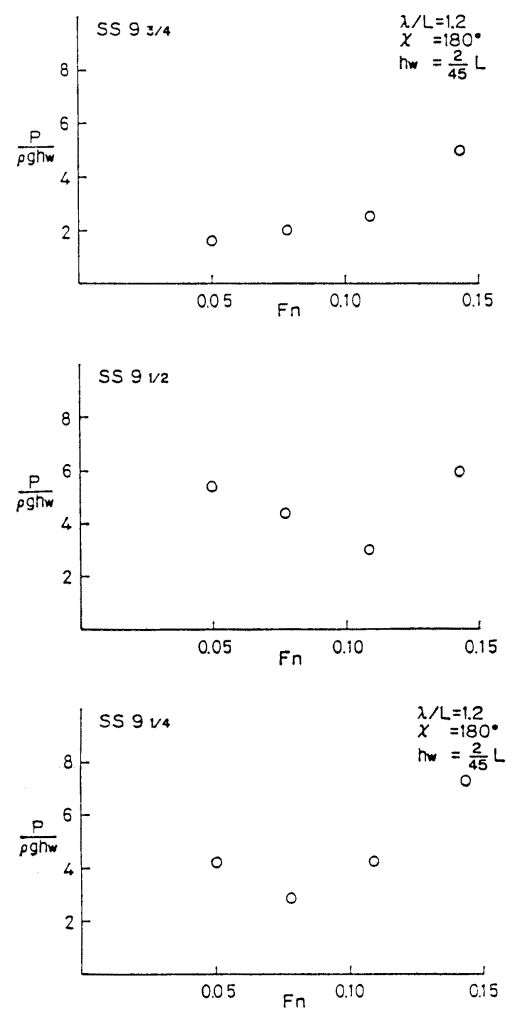

Fig. 16 Impact pressure versus Froude number $(\lambda / L=1.2)$

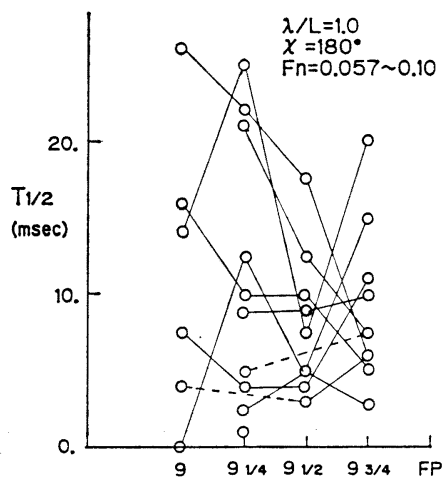

Fig. 17 Duration of the impact

in the figures. There is, however, slight discrepancies in the lags.

Next it will be discussed how the location of the waterline will be estimated by the OSM calculation. Figs. 20 and 21 show how long the waterline takes to move longitudinally. The estimation depicted by lines with circles show that the impact moves in much shorter time (faster) as approaching the bow. The experiment represented by solid symbols shows no such trend as is shown in the calculation. The difference presumably comes both from differences of phase appeared in Figs. 18 and 19 as well as from mechanisms which are neglected in 

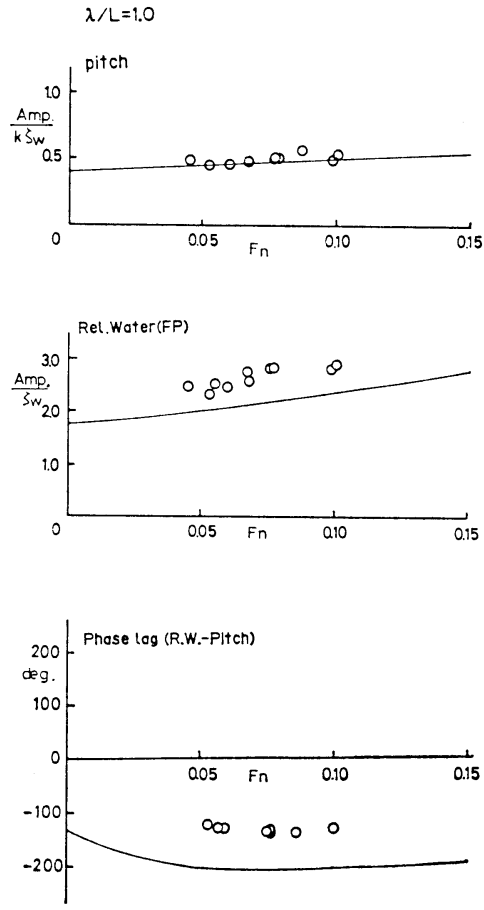

Fig. 18 Comparison of calculated ship motions with the experiment $(\lambda / L=1.0)$

$\lambda / L=1.2$
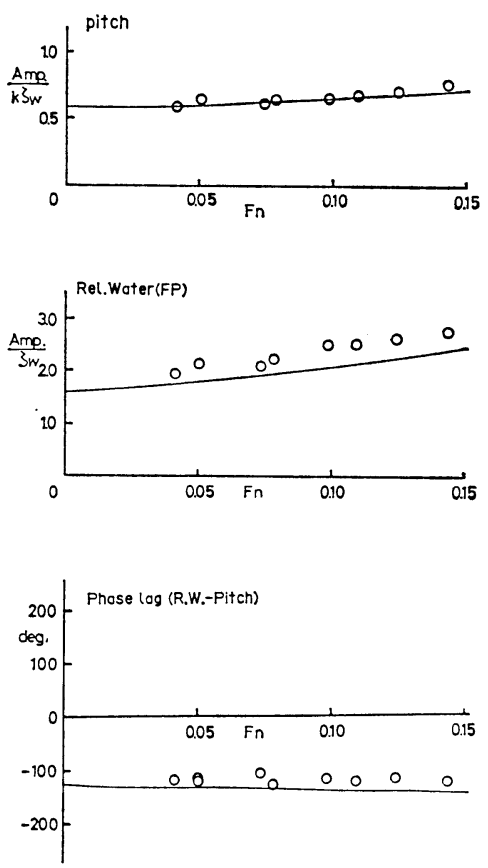

Fig. 19 Comparison of calculated ship motions with the experiment $(\lambda / L=1.2)$

the OSM calculation.

Figs. 22 and 23 show the estimation of the peak impact pressure, together with the behaviors of

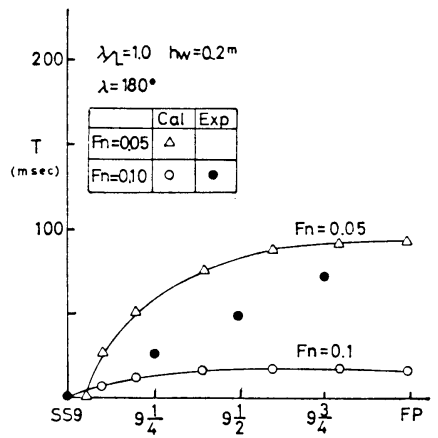

Fig. 20 Calculation of time lag in the occurrence of the impact $(\lambda / L=1.0)$

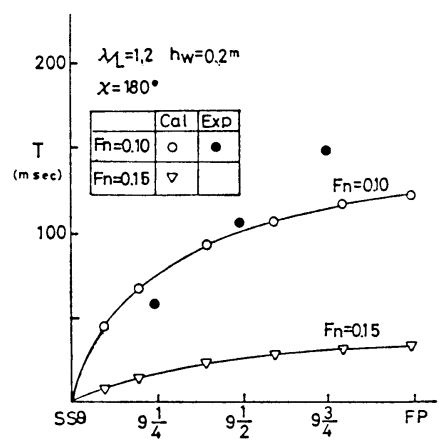

Fig. 21 Calculation of time lag in the occurrence of the impact $(\lambda / L=1.2)$

the vertical impact velocity $\left(V_{r}\right)$ and the relative inclination $\left(\theta_{r}\right)$ between the bottom and the wave surface. It is seen that the impact pressure Decomes very high when $\theta_{r}$ approaches zero. It is also seen that the estimation gives fairly larger value than the experiment which is shown in the Figs. 15 and 16.

It will be duscussed in the following why large discrepancy occurs between the estimation and the experiment. The difference comes either from the estimation formula of the impact pressure or the estimation of the ship motion, especially of the location of the water line. We have seen that the estimation of the time lag of the waterline differ appreciably from the measurement. Since the travelling speed contributes to the impact pressure substantially, it may be worth while to see what happens if we estimate the pressure with the measured travelling velocity of the waterline $\left(U_{r}\right)$, while keeping the present formula for the pressure. Here we define as $U_{r}=V_{r} \cot \theta$. Figs. 24 and 25 show its results together with the measured peak values. The vertical axis indicates the estimated values and the horizontal axis shows the measurements. The peak values are nondimensionalized with respect to the water head de- 

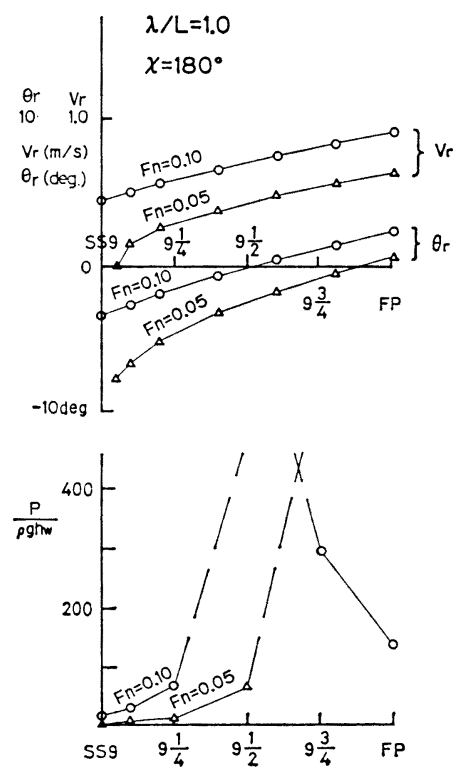

Fig. 22 Calculation of vertical velocity, local inclination and peak pressure $(\lambda / L=1.0)$
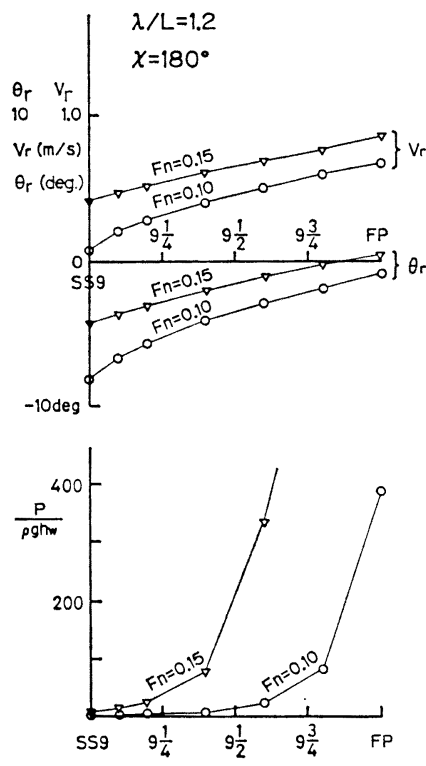

Fig. 23 Calculation of vertical velocity, local inclination and peak pressure $(\lambda / L=1.2)$

fined by the wave amplitude $\left(\zeta_{w}\right)$. It is seen that the estimation represented by circles has been concentrated on the diagonal straight line, which means this estimation based upon the measured travelling velocity agrees well the measured wave impact pressure. This tells us that the poor agreement in the impact pressure estimation can be attributed to the inaccuracy of OSM in estimating

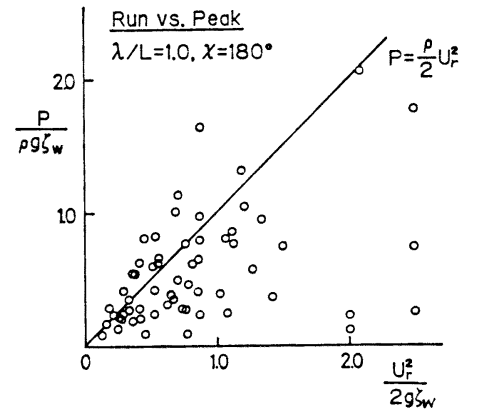

Fig. 24 Comparison of measured impact pressure and the estimation by using the measured running velocity $(\lambda / L=1.0)$

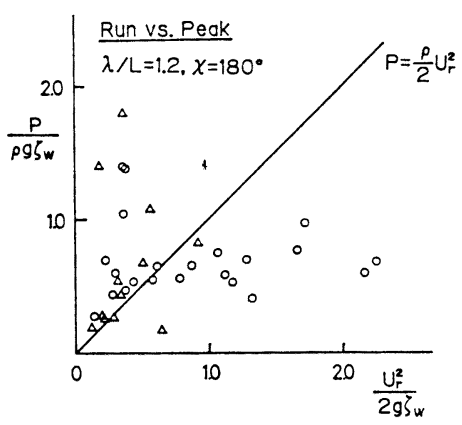

Fig. 25 Comparison of measured impact pressure and the estimation by using the measured running velocity $(\lambda / L=1.2)$

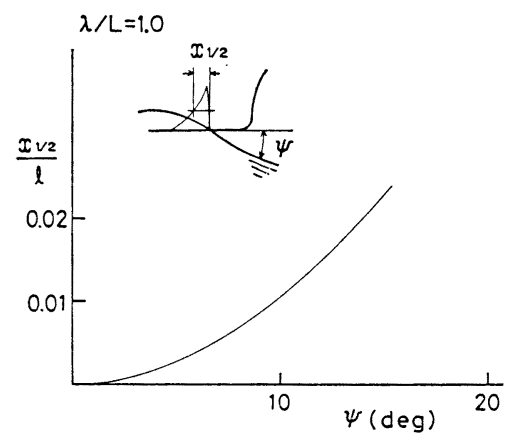

Fig. $26 X_{1 / 2}$ versus inclination

the location of the waterline and thus the agreement can be improved by refining the ship motion estimation while keeping the formula given here.

It will be examined in the last how well the present formula estimates the temporal and spatial variation. Fig. 26 shows the estimation by the present theory of the variation of $X_{1 / 2}$ on the bottom centerline to the inclination. The ratio of $X_{1 / 2}$ to the waterline length $(l)$ increases as the angle is increased. The typical value is about 0.01 for $5 \mathrm{deg}$. of inclination if the measured ship motion is input. 
On the other hand, $X_{1 / 2}$ be estimated from the measured $T_{1 / 2}$ and the travelling velocity of the waterline in an approximate way $X_{1 / 2}=U_{r} T_{1 / 2}$ according to the present theory. Typical value is 5 to $10 \mathrm{msec}$ for $T_{1 / 2}$ and $5 \mathrm{~m} / \mathrm{sec}$ for $U_{r}$. Thus we have

$$
X_{1 / 2}=500 \times 0.01=5(\mathrm{~cm}) \text {. }
$$

Since the waterline length $(l)$ of the model is about $4.3 \mathrm{~m}$, we have

$$
X_{1 / 2} / l=0.012 \text {. }
$$

The agreement tells us the present calculation is able to explain the spatial and temporal variation of the pressure.

\section{Conclusion}

A new calculation for the wave impact pressure over the ship hull has been presented and its numerical results have been compared with experiments. Conclusions are

(1) This method can tell effects of the longitudinal flow upon the wave impact pressure and give rational formulation of wave impact pressure over the inclined flat bottom, which was not possible for the conventional method.

(2) Effects of ship motion and the incoming wave to the impact pressure can be taken into account through relative wave velocity and the instantaneous local inclination with respect to wave surface.

(3) Present composite solution is in fair agreement with experiment qualitatively.

(4) Measured values of peak pressure are lower than the estimated by present method.

(5) The reason of the discrepancies can be attributed to inadequate accuracy in estimating the ship motions.

(6) The agreement between calculation and the experiment can be improved considerably if we utilize present formula with the measured ship motion data instead of the calculated values by OSM.

(7) Spatial and temporal variation of the wave impact pressure can be explained well by the present theory.

\section{Acknowledgement}

The author wishes to express sincere thanks to Prof. M. Fujino of University of Tokyo for his invaluable suggestions and guidances in preparing this work.

\section{References}

1) von Kármán, Th.: "The Impact of Seaplanes Float During Landing," NACA TN 321, Oct. (1929), pp. 1-8.

2) Wagner, H.: "Uber Stoss- und Gleitvorgange an der oberflacke von Flussigkeiten," ZAMM, band 4, heft 4, (1932), pp. 193-235.

3) Ochi, M. K. and Motter, L.E.: "A Method to Estimate Slamming Characteristics for Ship Design," Marine Technology, Vol.8, No. 2 (1971), pp. 219-232.

4) Stavovy, A. B. and Chuang, S.-L.: "Analytical Determination of Slamming Pressures for High-speed Vehicles in Waves," Journal of Ship Research, Vol.20, No. 4 (1976), pp. 190-198.

5) Chuang, S.-L.: "Theoretical Investigations on Slamming of Cone-Shaped Bodies," Journal of Ship Research, Vol.13, No. 4 (1969), pp. 276-283.

6) Watanabe, I.: "Analytical Expression of $\mathrm{Hy}$ drodynamic Impact Pressure by Matched Asymptotic Expansion Technique," Transaction of the West-Japan Society of Naval Architects, No. 71, March (1986), pp. 77-85.

7) Watanabe, I.: "On Hydrodynamic Impact Pressure Acting upon Flat Bottomed Ships," (in Japanese) Journal of the Society of $\mathrm{Na}$ val Architects of Japan, No.159, June (1986), pp. 201-210.

8) Watanabe, I.: "Theoretical Investigation on the Wave Impact Loads on Ships," presented at the 16 th Symposium on Naval Hydrodynamics in Berkeley, July (1986).

9) Cole, J. D.: "Perturbation Methods in Applied Mathematics," Blaisdell (1968).

10) Milne-Thomson, L. M.: "Theoretical Hydrodynamics," Macmillan, (1955).

11) Sedov, L. I.: "Two-dimensional Problems in Hydrodynamics and Aerodynamics," Interscience, (1950).

12) Kochin, N. K., Kibel, I. A. and Roze, N. V.: "Theoretical Hydromechanics," Interscience, (1955).

13) Abramowitz, M. and Stegun, I. A.: "Handbook of Mathematical Functions," Dover, (1970). 